\title{
To Sleep, Perchance to Dream: Acute and Chronic Sleep Deprivation in Acute Care
}

\section{Surgeons}

Jamie J Coleman, MD, FACS ${ }^{1}$, Caitlin K Robinson, BS ${ }^{1}$, Ben L Zarzaur, MD, MPH, FACS ${ }^{2}$, Lava Timsina, PhD, $\mathrm{MPH}^{2}$, Grace S Rozycki, MD, MBA, FACS ${ }^{3}$, David V Feliciano, MD, FACS $^{4}$

${ }^{1}$ Denver Health Medical Center, Denver, CO

${ }^{2}$ Department of Surgery, Indiana University School of Medicine, Indianapolis, IN

${ }^{3}$ Department of Surgery, Johns Hopkins University School of Medicine, Baltimore, MD

${ }^{4}$ Department of Surgery, University of Maryland School of Medicine, Baltimore, MD

\section{Disclosure Information: Nothing to disclose.}

Presented at the $77^{\text {th }}$ Annual Meeting of the American Association for the Surgery of Trauma, San Diego, California, September 2018.

Corresponding author:

Dr Jamie J Coleman, MD, FACS

Denver Health Medical Center

Surgery

777 Bannock St

MC 0206

Denver, Colorado 80204

UNITED STATES

3036021845

mobile: 3129333029

FAX: 3036021830

jiones79@gmail.com;jamie.coleman@dhha.org)

Brief Title: Acute Care Surgeons and Sleep Deprivation

This is the author's manuscript of the article published in final edited form as:

Coleman, J. J., Robinson, C. K., Zarzaur, B. L., Timsina, L., Rozycki, G. S., \& Feliciano, D. V. (2019). To Sleep, Perchance to Dream: Acute and Chronic Sleep Deprivation in Acute Care Surgeons. Journal of the American College of Surgeons. https://doi.org/10.1016/j.jamcollsurg.2019.03.019 


\section{ABSTRACT}

Background: Acute and chronic sleep deprivation are significantly associated with depressive symptoms and felt to be contributors to the development of burnout. In-house call (IHC) inherently includes frequent periods of disrupted sleep and is common amongst acute care surgeons (ACS). The relationship between IHC and sleep deprivation (SD) amongst ACS has not been previously studied. The goal of this study was to determine prevalence and patterns of SD in ACS.

Study Design: A prospective study of ACS with IHC responsibilities from two Level I trauma centers was performed. Participants wore a sleep tracking device continuously over a 3-month period. Data collected included age, gender, schedule of IHC, hours and pattern of each sleep stage (light, slow wave (SWS), and REM), and total hours of sleep. Sleep patterns were analyzed for each night excluding IHC and categorized as normal $(\mathrm{N})$, acute sleep deprivation (ASD), or chronic sleep deprivation (CSD). Results: 1421 nights were recorded amongst 17 ACS. (35.3\% female; ages 37-65, mean 45.5 years). Excluding IHC, average amount of sleep was 6.54 hours with $64.8 \%$ of sleep patterns categorized as ASD or CSD. Average amount of sleep was significantly higher on post-call day 1 (6.96 hours, $\mathrm{p}=0.0016)$, but decreased significantly on post-call day 2 (6.33 hours, $\mathrm{p}=0.0006)$. Sleep patterns with ASD and CSD peaked on post-call day 2 , and returned to baseline on post-call day $3(\mathrm{p}=0.046)$.

Conclusion: Sleep patterns consistent with ASD and CSD are common amongst ACS and worsen on postcall day 2. Baseline sleep patterns were not recovered until post-call day 3. Future study is needed to identify factors which impact physiologic recovery after IHC and further elucidate the relationship between SD and burnout.

Keywords: sleep deprivation, burnout, acute care surgeon, trauma surgeon 


\section{Background}

Sleep deprivation leads to a significant number of physical and emotional consequences including increased risk of stroke, obesity, colorectal cancer, breast cancer, suicide, depression and low quality of life scores (1-3). Mood alterations following periods of sleep deprivation specifically include the exacerbation of negative emotions and a reduction in feelings of empathy, both of which are key components of burnout (4-6). Burnout is a syndrome which is characterized by emotional exhaustion, a sense of depersonalization, and a decreased sense of accomplishment, particularly in relation to one's profession (7-9). Burnout, which is one of the chief contributors to depression and suicide is estimated to be as high as 50\% among surgeons in the United States (7). Studies since 2009 have shown between 28-40\% of surgeons surveyed experiencing symptoms of burnout, with each subsequent study reporting higher rates than those prior (8-11).

In this context, it is not surprising the number of hours worked and number of nights on call per week have a strong association with the development of burnout $(7,11,12)$. Compared to other surgical specialties, trauma surgeons reported the highest workload, with one study reporting $65 \%$ of trauma surgeons surveyed having worked $>70$ hours/week (11). Trauma and acute care surgeons (ACS) surgeons, along with cardiovascular, transplant, and urology report not only the longest hours but the most nights on call (12). Prior surveys, however, have not distinguished between nights of home versus in-house call $(5,12)$. In-house call for attending surgeons, which is commonly required on trauma and acute care surgery services, inherently includes frequent periods of disrupted sleep(13).

Sleep deprivation can occur as a result of either a lack of quality or quantity of sleep obtained. Acute sleep deprivation is defined by periods of wakefulness that extend beyond 16-18 hours while chronic sleep deprivation occurs when too little or poor quality sleep is achieved over multiple nights (14). Sleep deprivation can also occur as a function of loss of either REM 
(rapid eye movement) or slow wave sleep - two main stages of sleep (15). Loss of REM sleep has been associated with decreased ability of perceptual learning and memory consolidation, while loss of slow wave sleep reduces the ability to perform simple tasks (15). Sleep deprivation, burnout, and depression have clear negative side effects not only for the surgeon but also for their patients. Acute and chronic sleep loss has been associated with an increase in medical errors, with one study reporting that burnout and depression were among the strongest contributors to major medical errors $(9,10,16)$.

There is a paucity of literature in regards to the prevalence of sleep deprivation amongst surgeons, particularly those specializing in trauma and acute care. In addition, the physiologic impact of in-house call on sleep and the amount of time that is needed to recover after nights of in-house call have not been previously studied. The purpose of this study was to characterize patterns of sleep and recovery surrounding nights of in-house call and to determine the prevalence of sleep deprivation amongst trauma and acute care surgeons with in-house call responsibilities.

$\underline{\text { Methods }}$

After approval by the Institutional Review Board of Indiana University School of Medicine, a prospective observational study of acute care surgeons with in-house call responsibilities was performed at Indiana University Health Methodist Hospital and Sydney and Lois Eskenazi Hospital over a period of three months. These are both American College of Surgeons verified Level I trauma centers in Indianapolis, Indiana. Attending trauma or acute care surgeons with in-house call responsibilities were eligible for enrollment. There were no exclusion criteria within this cohort. Acute care surgeons who volunteered to participate in the study were given a fitness and sleep tracking device called Whoop!. The surgeons then 
downloaded the accompanying smart phone application and created a deidentified profile. The participants were instructed to wear the device either on the wrist or upper arm continuously for three months. They also were required to log into the smart phone application once daily to ensure non-interrupted data collection on call and non-call nights.

The Whoop! TM device is a wearable biometric instrument, manufactured by the company that continuously measures physiologic stress and the body's response to that stress. It measures heart rate, heart rate variability, ambient temperature, skin conductivity and movement via a 3axis accelerometer 100 times per second on a continual basis. Through heart rate, heart rate variability, and movement detection, it automatically detects sleep and sleep stage. Whoop! TM has been internally validated in a laboratory setting by comparison against graded polysomnography conducted by a certified polysomnography technologist. Whoop! TM was shown to have $96 \%$ accuracy, $93 \%$ sensitivity, $98 \%$ specificity. Data collected included age, gender, hours and pattern of each sleep stage, and total hours of sleep. Sleep stages were recorded as light, slow wave, or rapid eye movement (REM). Sleep patterns were also analyzed for each night excluding nights of in-house call. In-house call night patterns were unable to be analyzed secondary to either complete lack of sleep or clearly disrupted sleep patterns.

Normal sleep is comprised of two different physiologic states, rapid eye movement (REM) sleep and non-REM sleep (17). Non-REM sleep is then further divided into four stages, with stages 1 and 2 collectively referred to as light sleep and stages 3 and 4 as slow wave sleep (17). REM sleep normally constitutes approximately $20-25 \%$ of total sleep time, with slow wave sleep occupying another 13-23\%, and light sleep occupying the remainder (17). A normal sleep pattern in a healthy, rested adult begins with non-REM sleep and includes progression to slow wave sleep for approximately 80 - 100 minutes before the first episode of REM sleep (17). After 
this first cycle of REM sleep, non-REM and REM sleep then cycle throughout the sleep period, with slow wave sleep decreasing in duration and frequency and REM sleep increasing in duration and frequency as the night progresses (17). In people with one or two nights of decreased sleep, this normal pattern of sleep is characteristically altered (17). Recovery from acute sleep deprivation is marked by a predominance of slow wave sleep and a delay in or the prevention of REM sleep (14). In this study, nights of sleep with two or more cycles of slow wave sleep prior to a cycle of REM sleep were classified as consistent categorized as acute sleep deprivation. Chronic sleep restriction and irregular sleep schedules also show a characteristic sleep pattern which consists of premature REM sleep (14). Nights of sleep with one or more episodes of REM prior to the onset of slow wave sleep were thusly categorized as consistent with chronic sleep deprivation.

Descriptive analysis using chi-square test followed by tests of proportions for sleep patterns (normal, acute, or chronic) was performed to compare difference in proportions between pre-call and post-calls consecutively at day 1 through 3 . For circumstances in which participants took q2, or every other night call, the data was recorded as post-call day 1 data, not pre-call data. Specifically, when a date was both post-call day 1 and pre-call due to the every other night schedule, the data was used and analyzed as post-call day 1 data only, not as pre-call. Within this study period, there were 23 instances of every other night call amongst 10 of the participants. Pairwise comparisons were made using Wald tests for the linear relationship after estimation of the proportion. For continuous variables, such as total sleep hours, REM sleep hours, and slow wave sleep hours, Paired Student's t-tests were used to examine the mean differences between pre-call and post-calls consecutively at day 1 through 3 . All tests were examined at 0.05 level of 
significance, and 95\% confidence intervals were reported. Stata/SE 14.2[TLR1] (StataCorp.

College Station, TX) was used as software for the analysis.

$\underline{\text { Results }}$

There were 19 surgeons who met the inclusion criteria of in-house call responsibilities at either Indiana University Health Methodist Hospital or Sydney and Lois Eskenazi Hospital, and were approached to volunteer for the study. Eighteen surgeons consented to participate, with one surgeon terminating participation prior to the conclusion of the trial, secondary to noncompliance of device wear. A total of 1421 nights, including 227 nights of call, were recorded amongst the remaining 17 participants. The majority of participants were male $(n=11,64.7 \%)$, with a mean age of 45.5 years (range 37-65). Average sleep latency (time it takes to fall asleep) across all nights included in analysis was 4 minutes and 37 second.

The average number of in-house call nights per month during the study period was $2.5-6$, with an overall average of 4.85 calls per surgeon. Excluding nights of in-house call, the average amount of sleep obtained by participants was 6.54 hours per night. Participants slept an average of 6.45 hours on the nights before call, and slept a significantly higher number of hours, 6.96, on post call day $1(\mathrm{p}<0.001)$. From post-call day 1 , the number of sleep hours decreased significantly to an average of $6.33(\mathrm{p}<0.001)$. Hours slept on post-call day 3 increased to 6.65 , however no significance was found in comparison to number of hours slept on nights before call or post-call day 2 (Figure 1, Table 1).

Excluding nights of in-house call, sleep patterns were categorized as consistent with either normal, acute or chronic sleep deprivation. Nights of sleep with one or more episodes of REM prior to the onset of slow wave sleep were categorized as chronic sleep deprivation. Acute sleep deprivation was defined by nights of sleep with two or more cycles of slow wave sleep 
prior to a cycle of REM sleep. Sleep patterns consistent with either acute or chronic sleep deprivation were recorded on $64.8 \%$ of nights. Nights of in-house call were not able to be analyzed due to the wide range of variability in the quality and pattern of sleep due to frequent disruptions and at times, complete lack of sleep.

Participants obtained an average of 1.12 hours of REM sleep and 1.24 hours of slow wave sleep on nights preceding in-house call. On post-call day 1 , the number of REM sleep hours significantly increased to $1.32(\mathrm{p}=0.02)$ and there was an increase in the amount of slow wave sleep to 1.38 hours but the difference did not reach statistical significance $(p=0.07)$. The amount of REM sleep decreased on post-call day 2 to 1.15 hours and then increased to 1.24 hours on post-call day 3 although statistical significance was not reached. Slow wave sleep significantly decreased on post-call day 2 to 1.11 hours $(\mathrm{p}<0.001)$, then increased to 1.28 hours on post-call day 3 - however, the increase on post-call day 3 did not reach statistical significance. (Figure 2, Table 2).

The percentage of nights with patterns consistent with acute sleep deprivation decreased from $44.0 \%$ on post-call day 2 to $40.3 \%$ on post-call day 3 , but did not reach significance. Sleep patterns categorized as chronic sleep deprivation decreased from $25.8 \%$ post-call day 2 to $18.6 \%$ on post-call day 3 , but also did not reach significance. Normal sleep patterns were recorded in $36.8 \%$ of pre-call nights and were not statistically different from post-call day 1 patterns, in which 37.0\% were normal (Figure 3, Table 3). The percentage of normal sleep patterns decreased to $29.8 \%$ on post-call day 2 and significantly increased to $41.1 \%$ on post-call day 3 $(\mathrm{p}<.04)$

\section{Discussion}


Work-related stress is a significant contributor to the development of both burnout and depression. For surgeons in particular, there are unique physical and psychological components that contribute to stress in the workplace. The act of performing surgery, participation in trauma activations, the leading of difficult conversations, and sleep deprivation have all been shown to produce physiologic changes in heart rate and heart rate variability that are consistent with moderate to high stress levels (18-21). Given that information, it is not surprising that a career in surgery is associated with burnout rates reported in the range from $40-50 \%(7,19,20,22)$. A 2008 survey conducted by the American College of Surgeons examining the presence of distress and career satisfaction amongst 14 surgical specialties, however, revealed that not all surgeons and specialties experience burnout equally (11). In fact, trauma surgeons reported the lowest mental quality of life and highest incidence of burnout amongst the 14 specialties surveyed (11).

This study was therefore designed to physiologically track the sleep patterns and determine the prevalence of sleep deprivation within trauma and acute care surgeons at two level one trauma centers to begin addressing the rapidly expanding rates of burnout and depression in this professional field. In this study of acute care surgeons with in-house call responsibilities, the average amount of sleep recorded on non-call nights, 6.54 hours, is significantly less than the recommended amount of sleep. Sleep patterns consistent with either acute or chronic sleep deprivation were common amongst participants. Chronic sleep deprivation patterns, as well as the lowest amounts of REM and slow wave sleep were the most prevalent on post-call day 2 , with normal sleep patterns occurring at the highest frequency on post-call day 3 . These findings are similar to those discussed by Banks et al which suggest that 2 to 3 nights of extended sleep may be needed to return to baseline neurobehavioral functions $(14,23)$. 
To achieve the full restorative effects of sleep, sleep must be adequate in duration, continuity, and type $(14,23)$. Therefore, the right amount of sleep and the right quality of sleep are imperative in achieving physiologic recovery. Within this small study population, restoration of baseline sleep patterns was achieved on post-call day 3. Sleep deprivation can occur when either the amount or quality of sleep is poor and is self-reported to occur in $20 \%$ of American adults (14). In the present study, this population of attending trauma and acute care surgeons with in-house call responsibilities experience both forms of sleep deprivation, with almost half of post-call day 1 nights showing patterns of acute sleep deprivation.

Acute sleep deprivation is defined by periods of wakefulness that extend beyond 16-18 hours (14). A single episode of sleep deprivation has been shown to result in impairment of memory and response time, reduced threshold for stress, and decrease in constructive thinking, and cognitive processing speed (24-26). Chronic sleep deprivation occurs when too little or poor quality sleep is achieved over multiple nights. It is associated with neurobehavioral alterations as in acute sleep deprivation, as well as an increased risk of cardiovascular disease, hypertension, obesity and type 2 diabetes mellitus (27-29). Also, elevated cortisol levels and changes in immune function via natural killer cell activity and lymphokine-activated killer cell activity have been demonstrated in people with a sleep debt $(30,31)$. In addition, an elevated mortality risk has been found in adults reporting less than 6.5 hours of sleep per night (32).

Wakefulness and sleep are highly regulated brain activities, and a balance between REM and non-REM sleep is also crucial to optimal neurobehavioral performance, learning, memory, and daily functioning $(1,17,33,34)$. Typically, young, healthy adults should spend 7-9 hours a night sleeping with $20-25 \%$ or $1.40-2.25$ hours spent in REM sleep (13). Slow-wave sleep typically comprises $13-23 \%$ ( 0.9 to 2.1 hours) of sleep time. In comparison to results of this 
study, participants ranged from 17.1-19\% of sleep spent in REM and 17.0-19.8\% of sleep spent in slow wave.

In addition to the health consequences of sleep deprivation, a lack of sleep has been shown to impact performance in the workplace. Outside of the medical field, impaired and shortened sleep is one of the most common causes of accidents in industry and transportation $(35,36)$. An increase in sleep deprivation has been shown to lead to higher levels of stress and fatigue and is associated with lower levels of situational awareness, thus leading to workplace accidents which cost billions of dollars annually(1). The detrimental effects of sleep deprivation on cognitive performance have been compared to the effects of acute alcohol intoxication. A 17 hour period of wakefulness produces a decrease in performance equivalent to a blood alcohol level of $0.05 \%$, and a full 24 hours without sleep is similar to legal intoxication with a blood alcohol level of $0.10 \%(37-39)$.

Another indicator of sleep deprivation is sleep latency, which is defined as the amount of time it takes to fall asleep. This study population had an average sleep latency time of 4 minutes, 37 seconds which is widely considered to be pathologic and a symptom of behaviorally induced insufficient sleep syndrome (BIISS) (40, 41). Typically measured by the Multiple Sleep Latency Test (MSLT), latency time of $<5$ minutes is known to correlate with several pathologic sleep disorders, including narcolepsy $(40,42)$. Given the high rates of sleep deprivation recorded in this study, it is likely that this short sleep latency time is due to BIISS, rather than narcolepsy or another sleep disorder.

How performance changes from sleep deprivation translate into the realm of surgery is unclear, although it has been shown that restricted sleep can result in significant neurocognitive deficits, such as executive processing, sustained attention, and long-term memory (43). 
Interestingly, Mohtashami et al evaluated laparoscopic tasks performed in a simulator by nine gynecologists in both a sleep deprived ( $<3$ hours of sleep in prior 24 hours) and a legally intoxicated (>0.08 mg/ml) state (44). For easy tasks, the efficiency and errors rating were similar between the sleep deprived and intoxicated states. For complex tasks, however, the errors were significantly higher when the gynecologists were intoxicated as compared to when they were sleep deprived. Gerdes et al examined the effect of fatigue on both surgical residents and attending surgeons in a simulation setting and found an increase in error rates associated with post-call status (45). Cognitive error rates for both attending surgeons and residents increased post-call, but to a significantly lesser degree amongst the former. Differences shown in simulation have not as yet translated into differences in patient outcomes. Govindarajan et al examined the outcomes of patients who underwent procedures during the day performed by attending surgeons who had treated patients between the prior midnight and 7 a.m. (46). This study, which included 38,978 patients, did not find an increase in the risk of adverse outcomes for patients being treated by physicians who worked the night before. Participants in the current study returned to baseline on post-call day 3 , which is an area of research that requires further study to determine if this is also associated with cognitive error rates when compared to post-call day 1 . Future studies hope to accomplish not only recommendations regarding frequency of inhouse call scheduling, but also tools to aid in physiologic and cognitive recovery following nights of call.

Although the relationship between sleep deprivation and patient outcomes is unclear, the relationship between sleep deprivation and mood has been extensively studied. Fatigued individuals show lower emotional empathy, less ability to regulate emotions, and an increase in negative affect $(5,47)$. Chronic sleep deprivation has been shown to increase the prevalence and 
risk of depression $(48,49)$. Although the rates of depression amongst American surgeons are similar to those of the general population, the rate of suicide is much higher (50).

Trauma and acute care surgeons are a unique population, even within surgical subspecialties, as the former report the highest number of work hours and call nights per week (11). Also, trauma and acute care surgeons are more likely to take in-house call as attending surgeons, predisposing them to irregular sleep patterns. In addition to the known stress caused by performing surgery, trauma and acute care surgeons routinely participate in additional stressful situations including trauma activations, patient resuscitations, and difficult conversations with patients and families $(18,51)$.

Limitations of the study include small sample size and a lack of knowledge regarding medical history, social habits amongst participants, and exclusion of call night sleep analysis. Participants were not asked to disclose their medical history or social habits, including medication or alcohol use. Medications such as benzodiazepines, tricyclic antidepressants, monoamine oxidase inhibitors, and some serotonin reuptake inhibitors are known to alter sleep patterns in such a way as to mimic either acute or chronic sleep deprivation. In addition, alcohol intake, especially within two hours of sleep onset, can increase slow wave and suppress REM sleep in such a way as to create a pattern of acute sleep deprivation. Caffeine intake, which can affect heart rate, was also required to be recorded by participants. Travel, especially across time zones, is also known to disrupt normal sleep patterns, yet travel activity was not recorded by participants. Call-night sleep patterns were extremely disrupted and overall were unable to be analyzed for distribution of REM vs. NREM sleep cycles. Whoop! measures REM vs. NREM sleep cycles through heart rate variability which has been shown to match sleep cycles. Ad 
In conclusion, acute care surgeons with in-house call responsibilities commonly exhibit sleep patterns consistent with sleep deprivation. Additional research into how surgeons with mixed coverage patterns, such as physicians and surgeons who take call from home, would add insight to the extra burden potentially faced by trauma and acute care surgeons who take inhouse call. Further study in a multicenter trial is needed to generalize the current data; further clarify the timeline of recovery after in-house call; identify factors which aid or hinder recovery; examine the impact of age, gender, and years of experience; and define the relationship between sleep deprivation and feelings of burnout. 


\section{References:}

1. Abrams RM. Sleep Deprivation. Obstet Gynecol Clin North Am. 2015;42(3):493-506.

2. Owens J, Adolescent Sleep Working G, Committee on A. Insufficient sleep in adolescents and young adults: an update on causes and consequences. Pediatrics. 2014;134(3):e921-32.

3. Bernert RA, Turvey CL, Conwell Y, Joiner TE, Jr. Association of poor subjective sleep quality with risk for death by suicide during a 10-year period: a longitudinal, population-based study of late life. JAMA Psychiatry. 2014;71(10):1129-37.

4. Deliens G, Stercq F, Mary A, et al. Impact of Acute Sleep Deprivation on Sarcasm Detection. PLoS One. 2015;10(11):e0140527.

5. Guadagni V, Burles F, Ferrara M, Iaria G. The effects of sleep deprivation on emotional empathy. J Sleep Res. 2014;23(6):657-63.

6. Babson KA, Feldner MT. Temporal relations between sleep problems and both traumatic event exposure and PTSD: a critical review of the empirical literature. J Anxiety Disord. 2010;24(1):1-15.

7. Dimou FM, Eckelbarger D, Riall TS. Surgeon Burnout: A Systematic Review. J Am Coll Surg. 2016;222(6):1230-9.

8. Pulcrano M, Evans SR, Sosin M. Quality of Life and Burnout Rates Across Surgical Specialties: A Systematic Review. JAMA surgery. 2016;151(10):970-8.

9. Shanafelt TD, Balch CM, Bechamps G, et al. Burnout and medical errors among American surgeons. Ann Surg. 2010;251(6):995-1000.

10. Balch CM, Shanafelt T. Combating stress and burnout in surgical practice: a review. Advances in surgery. 2010;44:29-47. 
11. Balch CM, Shanafelt TD, Sloan JA, et al. Distress and career satisfaction among 14 surgical specialties, comparing academic and private practice settings. Annals of surgery. 2011;254(4):558-68.

12. Balch CM, Shanafelt TD, Dyrbye L, et al. Surgeon distress as calibrated by hours worked and nights on call. Journal of the American College of Surgeons. 2010;211(5):609-19.

13. Daniel VT, Ingraham AM, Khubchandani JA, et al. Variations in the Delivery of Emergency General Surgery Care in the Era of Acute Care Surgery. Joint Commission journal on quality and patient safety / Joint Commission Resources. 2019;45(1):14-23.

14. Banks SD, J; Basner, M; Dinges DF. Sleep Deprivation. In: Kryger MH RT, Dement WC, editor. Principles and practice of sleep medicine. Sixth edition ed. Philadelphia, Pennsylvania: Elsevier; 2017. p. 49-55.

15. Sanches I, Teixeira F, dos Santos JM, Ferreira AJ. Effects of Acute Sleep Deprivation Resulting from Night Shift Work on Young Doctors. Acta medica portuguesa. 2015;28(4):45762.

16. Veasey S, Rosen R, Barzansky B, et al. Sleep loss and fatigue in residency training: a reappraisal. Jama. 2002;288(9):1116-24.

17. Carskadon M, Dement, WC. Normal human sleep: An overview. In: Kryger MH RT, Dement WC, editor. Principles and practice of sleep medicine. Sixth edition ed. Philadelphia, Pennsylvania: Elsevier; 2017. p. 15-24.

18. Joseph B, Parvaneh S, Swartz T, et al. Stress among surgical attending physicians and trainees: A quantitative assessment during trauma activation and emergency surgeries. The journal of trauma and acute care surgery. 2016;81(4):723-8. 
19. Rieger A, Stoll R, Kreuzfeld S, et al. Heart rate and heart rate variability as indirect markers of surgeons' intraoperative stress. Int Arch Occup Environ Health. 2014;87(2):165-74. 20. Jones KI, Amawi F, Bhalla A, et al. Assessing surgeon stress when operating using heart rate variability and the State Trait Anxiety Inventory: will surgery be the death of us? Colorectal Dis. $2015 ; 17(4): 335-41$.

21. Neufeld EV, Carney JJ, Dolezal BA, et al. Exploratory Study of Heart Rate Variability and Sleep among Emergency Medical Services Shift Workers. Prehosp Emerg Care. 2017;21(1):18-23.

22. Shanafelt TD, Balch CM, Bechamps GJ, et al. Burnout and career satisfaction among American surgeons. Annals of surgery. 2009;250(3):463-71.

23. Basner M, Rao H, Goel N, Dinges DF. Sleep deprivation and neurobehavioral dynamics. Curr Opin Neurobiol. 2013;23(5):854-63.

24. Durmer JS, Dinges DF. Neurocognitive consequences of sleep deprivation. Semin Neurol. 2005;25(1):117-29.

25. Banks S, Van Dongen HP, Maislin G, Dinges DF. Neurobehavioral dynamics following chronic sleep restriction: dose-response effects of one night for recovery. Sleep. 2010;33(8):1013-26.

26. Goel N, Rao H, Durmer JS, Dinges DF. Neurocognitive consequences of sleep deprivation. Semin Neurol. 2009;29(4):320-39.

27. Hsieh SD, Muto T, Murase T, et al. Association of short sleep duration with obesity, diabetes, fatty liver and behavioral factors in Japanese men. Intern Med. 2011;50(21):2499-502. 
28. Cappuccio FP, Cooper D, D'Elia L, et al. Sleep duration predicts cardiovascular outcomes: a systematic review and meta-analysis of prospective studies. Eur Heart J. 2011;32(12):1484-92.

29. Cappuccio FP, Taggart FM, Kandala NB, et al. Meta-analysis of short sleep duration and obesity in children and adults. Sleep. 2008;31(5):619-26.

30. Spiegel K, Leproult R, Van Cauter E. Impact of sleep debt on metabolic and endocrine function. Lancet (London, England). 1999;354(9188):1435-9.

31. Irwin M, McClintick J, Costlow C, et al. Partial night sleep deprivation reduces natural killer and cellular immune responses in humans. FASEB J. 1996;10(5):643-53.

32. Kripke DF, Garfinkel L, Wingard DL, et al. Mortality associated with sleep duration and insomnia. Arch Gen Psychiatry. 2002;59(2):131-6.

33. Vyazovskiy VV, Delogu A. NREM and REM Sleep: Complementary Roles in Recovery after Wakefulness. Neuroscientist. 2014;20(3):203-19.

34. Siegel J. Rapid eye movement sleep. In: Kryger MH RT, Dement WC, editor. Principles and practice of sleep medicine. Sixth edition ed. Philadelphia, Pennsylvania: Elsevier; 2017. p. 78-95.

35. Philip P, Akerstedt T. Transport and industrial safety, how are they affected by sleepiness and sleep restriction? Sleep Med Rev. 2006;10(5):347-56.

36. Folkard S, Lombardi DA, Tucker PT. Shiftwork: safety, sleepiness and sleep. Ind Health. 2005;43(1):20-3.

37. Dawson D, Reid K. Fatigue, alcohol and performance impairment. Nature. 1997;388(6639):235. 
38. Williamson AM, Feyer AM. Moderate sleep deprivation produces impairments in cognitive and motor performance equivalent to legally prescribed levels of alcohol intoxication. Occup Environ Med. 2000;57(10):649-55.

39. Arnedt JT, Wilde GJ, Munt PW, MacLean AW. How do prolonged wakefulness and alcohol compare in the decrements they produce on a simulated driving task? Accid Anal Prev. 2001;33(3):337-44.

40. Komada Y, Inoue Y, Hayashida K, et al. Clinical significance and correlates of behaviorally induced insufficient sleep syndrome. Sleep Med. 2008;9(8):851-6.

41. Goldbart A, Peppard P, Finn L, et al. Narcolepsy and predictors of positive MSLTs in the Wisconsin Sleep Cohort. Sleep. 2014;37(6):1043-51.

42. Sullivan SS, Kushida CA. Multiple sleep latency test and maintenance of wakefulness test. Chest. 2008;134(4):854-61.

43. Lowe CJ, Safati A, Hall PA. The neurocognitive consequences of sleep restriction: A meta-analytic review. Neurosci Biobehav Rev. 2017;80:586-604.

44. Mohtashami F, Thiele A, Karreman E, Thiel J. Comparing technical dexterity of sleepdeprived versus intoxicated surgeons. JSLS. 2014;18(4).

45. Gerdes J, Kahol K, Smith M, et al. Jack Barney award: the effect of fatigue on cognitive and psychomotor skills of trauma residents and attending surgeons. American journal of surgery. 2008;196(6):813-9; discussion 9-20.

46. Govindarajan A, Urbach DR, Kumar M, et al. Outcomes of Daytime Procedures Performed by Attending Surgeons after Night Work. The New England journal of medicine. 2015;373(9):845-53. 
47. Dinges DF, Pack F, Williams K, et al. Cumulative sleepiness, mood disturbance, and psychomotor vigilance performance decrements during a week of sleep restricted to 4-5 hours per night. Sleep. 1997;20(4):267-77.

48. Coulehan J, Williams PC. Vanquishing virtue: the impact of medical education. Acad Med. 2001;76(6):598-605.

49. Roberts RE, Duong HT. The prospective association between sleep deprivation and depression among adolescents. Sleep. 2014;37(2):239-44.

50. Shanafelt TD, Balch CM, Dyrbye L, et al. Special report: suicidal ideation among American surgeons. Arch Surg. 2011;146(1):54-62.

51. Brown R, Dunn S, Byrnes K, et al. Doctors' stress responses and poor communication performance in simulated bad-news consultations. Acad Med. 2009;84(11):1595-602. 
Figure Legend:

Figure 1. Overall trend of total hours of sleep, with significant decrease on post-call day 2 $(\mathrm{p}=0.0016)$.

Figure 2. Trend of hours spent in rapid eye movement (REM) and slow wave sleep, with lowest amount recorded on post call day $2($ REM $p=0.0767$; slow wave sleep $\mathrm{p}=0.0016)$

Figure 3. Percentage of acute sleep deprivation, chronic sleep deprivation, and normal patterns of sleep in relation to call. Lowest percentage of normal sleep patterns occurred on post-call day 2, with highest percentage recorded on post-call day $3(\mathrm{p}=0.0445)$ 
Table 1. Total Hours of Sleep

\begin{tabular}{|l|r|c|r|}
\hline Night as related to call night & Hours slept & $\begin{array}{c}\text { Hour difference (when } \\
\text { compared to) }\end{array}$ & p Value \\
\hline 1 Night Before Call & 6.45 & +0.09 (average) & $0.0016^{*}$ \\
\hline Post-call day 1 & 6.96 & +0.42 (night before call) & $.0006^{*}$ \\
\hline Post-call day 2 & 6.33 & -0.63 (post-call day 1) & .274 \\
\hline Post-call day 3 & 6.65 & +0.2 (night before call) & .1149 \\
\hline Post-call day 3 & 6.65 & +0.32 (post-call day 2 &
\end{tabular}

*Statistically significant 
Table 2. Total Hours of Rapid Eye Movement and Slow Wave Sleep

\begin{tabular}{|l|c|c|c|}
\hline $\begin{array}{l}\text { Night as related to call } \\
\text { night, sleep cycle }\end{array}$ & Hours & Hour difference & p Value \\
\hline 1 night before call & & & - \\
\hline REM & 1.12 & - & - \\
\hline Slow wave & 1.24 & - & $0.021^{*}$ \\
\hline $\begin{array}{l}\text { Post-call day 1 } \\
\text { as related to night before call }\end{array}$ & 1.32 & +0.20 & $0.0069^{*}$ \\
\hline REM & 1.38 & +0.14 & $0.007^{*}$ \\
\hline Slow wave & & & $0.0016^{*}$ \\
\hline Post-call day & 1.15 & +0.03 & \\
\hline REM & 1.11 & -0.13 & 0.87 \\
\hline Slow wave & & & 0.07 \\
\hline Post-call day 3 & 1.24 & +0.12 & \\
\hline REM & 1.28 & +0.04 & \\
\hline Slow wave & & & \\
\hline
\end{tabular}

*Statistically significant

REM, rapid eye movement 
Table 3. Percentages of sleep patterns present.

\begin{tabular}{|c|c|c|}
\hline $\begin{array}{l}\text { Type of sleep pattern, } \\
\text { post-call day }\end{array}$ & \% consistent with pattern & p Value \\
\hline Acute sleep deprivation & & 0.53 \\
\hline Post-call day 2 & 44.0 & \\
\hline Post-call day 3 & 40.2 & 0.14 \\
\hline Chronic sleep deprivation & & \\
\hline Post-call day 2 & 25.8 & \\
\hline Post-call day 3 & 18.6 & \\
\hline Normal sleep pattern & & \\
\hline Pre-call night & 36.8 & $0.0485^{*}$ \\
\hline Post-call day 1 & 37.0 & \\
\hline Normal sleep pattern & & \\
\hline Post-call day 2 & 29.8 & \\
\hline Post-call day 3 & 41.1 & \\
\hline
\end{tabular}

*Statistically significant 
Precis

Acute care surgeons with in-house call responsibilities commonly exhibit sleep patterns

consistent with acute and chronic sleep deprivation. These abnormal sleep patterns worsen on post-call day 2, with recovery to baseline achieved on post-call day 3 . 


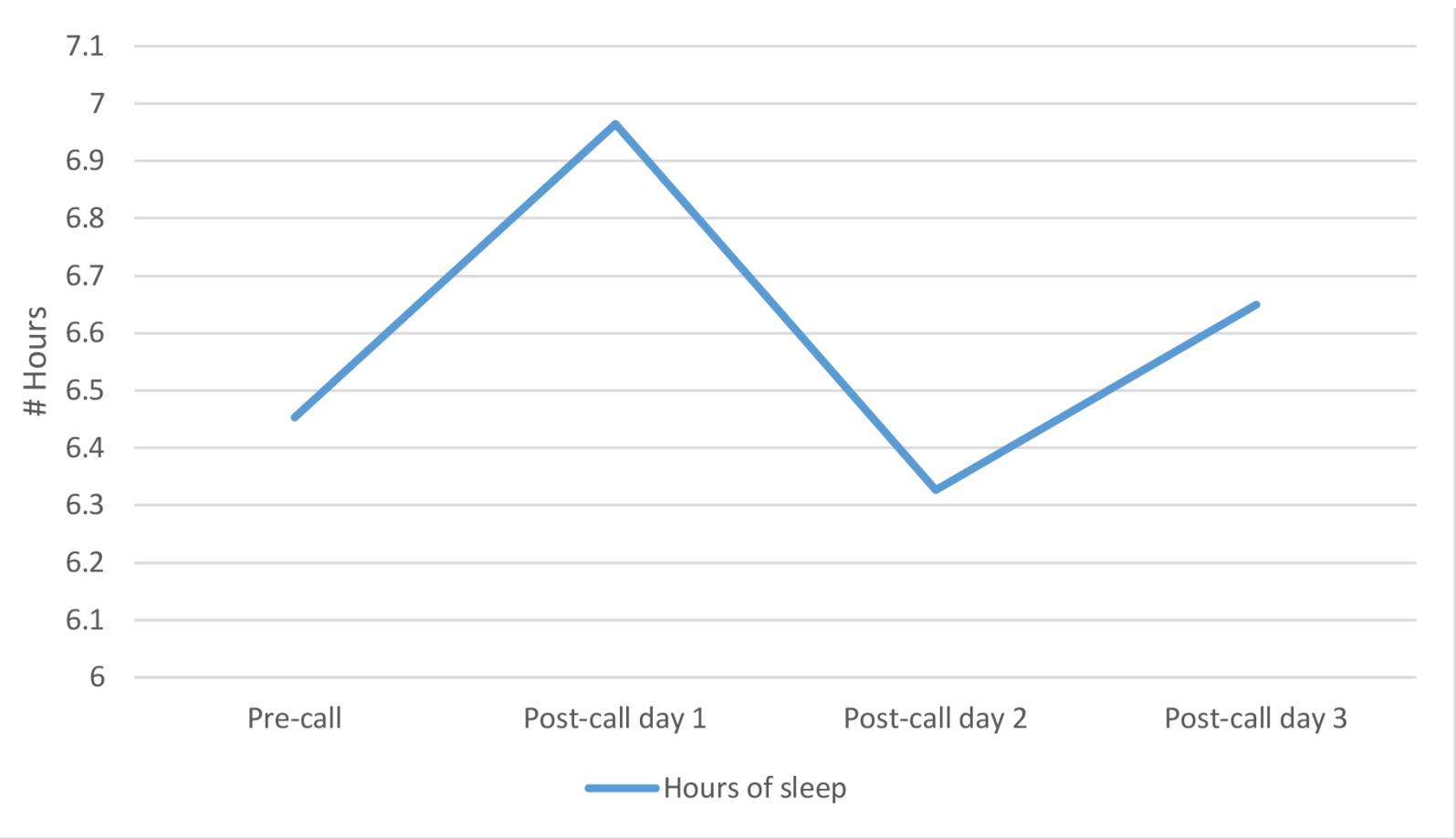




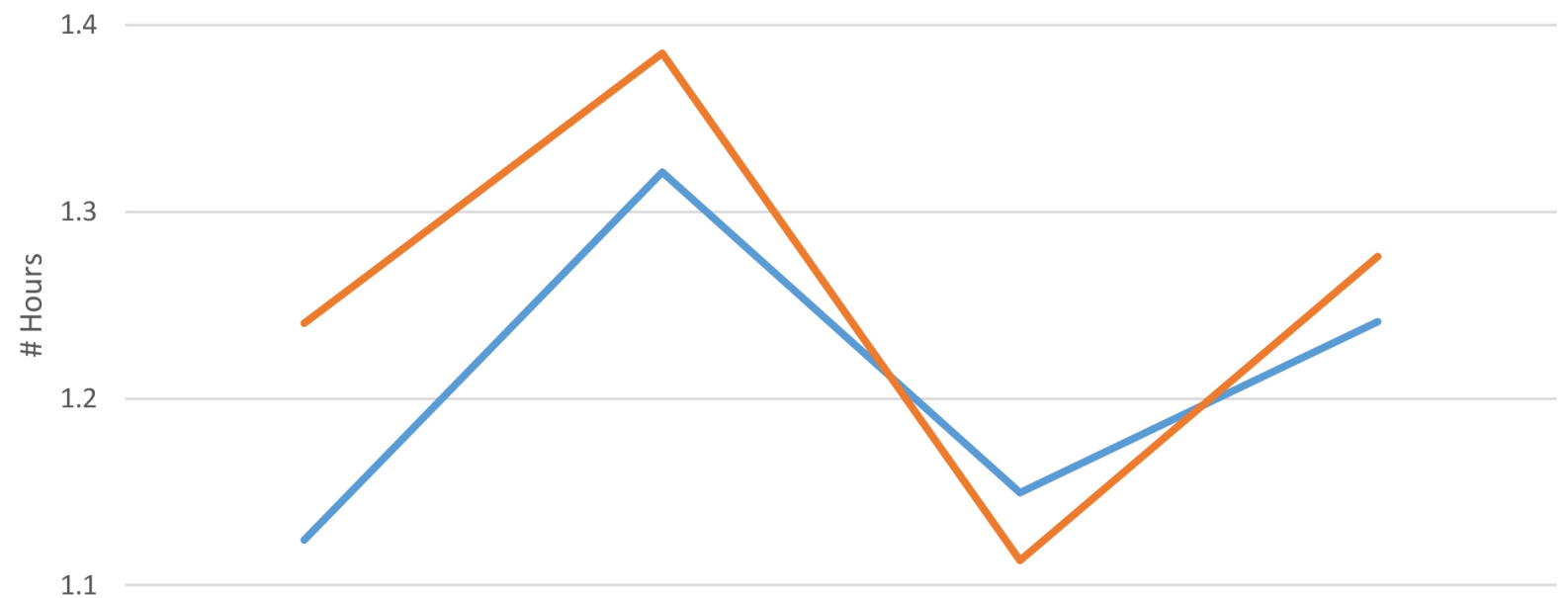

1.1

1

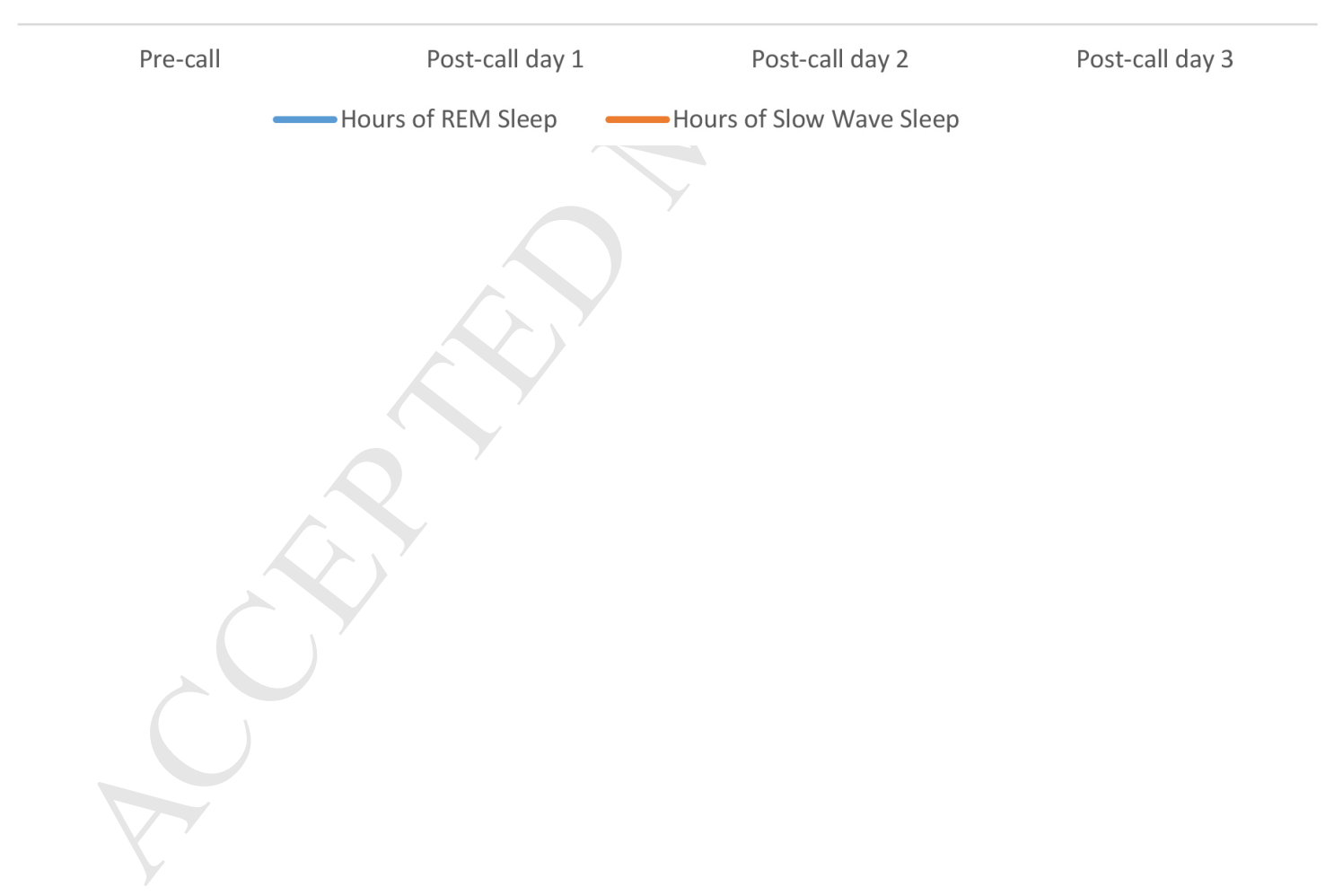




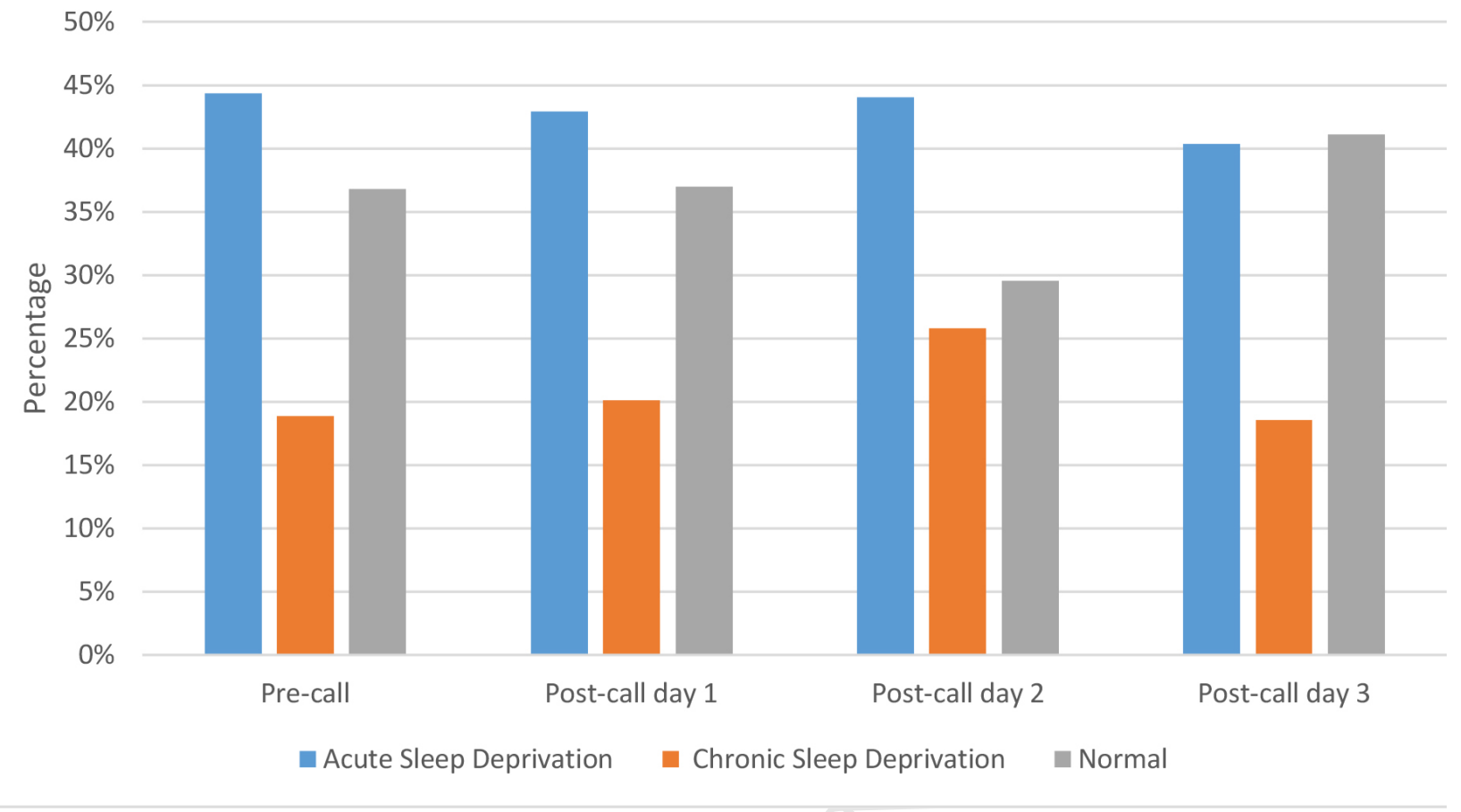

